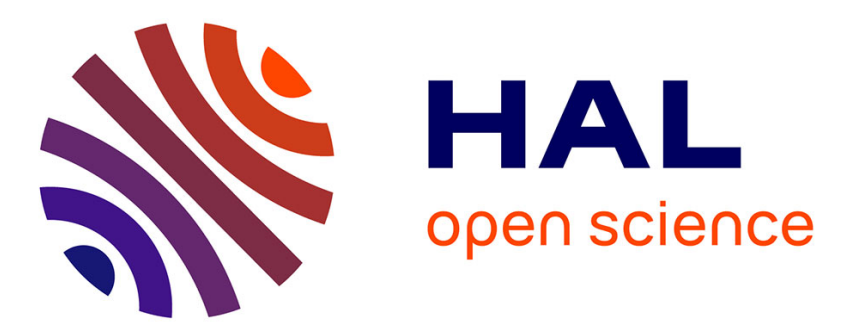

\title{
Impacts du changement climatique, sécurité hydrique et enjeux agricoles. Le cas de la Chine du nord
}

Nathalie Rousset

\section{To cite this version:}

Nathalie Rousset. Impacts du changement climatique, sécurité hydrique et enjeux agricoles. Le cas de la Chine du nord. Perspectives chinoises, 2007, 98, pp.32-41. halshs-00170103

\section{HAL Id: halshs-00170103 \\ https://shs.hal.science/halshs-00170103}

Submitted on 6 Sep 2007

HAL is a multi-disciplinary open access archive for the deposit and dissemination of scientific research documents, whether they are published or not. The documents may come from teaching and research institutions in France or abroad, or from public or private research centers.
L'archive ouverte pluridisciplinaire HAL, est destinée au dépôt et à la diffusion de documents scientifiques de niveau recherche, publiés ou non, émanant des établissements d'enseignement et de recherche français ou étrangers, des laboratoires publics ou privés. 


\section{NOTE DE TRAVAIL}

$N^{\circ} 20 / 2007$

\section{Impacts du changement climatique, sécurité hydrique et enjeux agricoles}

\section{Le cas de la Chine du nord}

\section{Nathalie Rousset}

avril 2007

\section{LEPII}





\title{
Impacts du changement climatique, sécurité hydrique et enjeux agricoles. Le cas de la Chine du nord
}

\author{
Nathalie Rousset \\ LEPII, Université de Grenoble, CNRS ; 38000 Grenoble
}

Résumé : Le changement climatique fait peser de nombreux risques d'impacts en Chine. Les ressources en eau dans les plaines du nord pourraient être particulièrement affectées. Si les équilibres sont déjà souvent rompus dans cette région, le réchauffement attendu jouera un rôle d'accélérateur de dynamiques existantes d'aggravation des déficits hydriques. Dans le contexte de compétition intersectorielle pour l'allocation de l'eau qui se dessine, la production céréalière du nord de la Chine apparaît comme un secteur particulièrement vulnérable. La diminution attendue des rendements des principales cultures pose alors la question des stratégies d'adaptation qui pourraient être adoptées. Mise en place de manière anticipée, une politique de l'eau axée de manière structurelle sur la rareté de la ressource permettrait de réduire ces risques tout en répondant à des défis déjà sensibles. Les impacts résiduels pourraient cependant rester conséquents. L'ouverture commerciale et les possibilités d'importer de l'eau sous forme de produits agricoles ouvrent de nouvelles perspectives pour une utilisation plus efficace des ressources hydriques,à mettre en balance avec le principe d'autosuffisance alimentaire.

La question du renforcement de l'effet de serre d'origine humaine porte les problèmes globaux d'environnement à un point jamais atteint dans l'histoire. Victime potentielle mais aussi responsable des bouleversements climatiques actuels, la Chine doit, de toute urgence, analyser les risques qu'elle encourt et définir les stratégies d'adaptation envisageables. L'objectif de cette contribution est de mettre en perspective les impacts potentiels du changement climatique sur les ressources hydriques et l'agriculture du nord de la Chine'.

L'utilisation du modèle MAGICC-SCENGEN² centré sur la Chine montre que les températures augmenteraient de 0,6 à $1,3^{\circ} \mathrm{C}$ d'ici 2030 et de 1,2 à $2,3^{\circ} \mathrm{C}$ en 2050 . Dans une telle éventualité, le nord du pays se réchaufferait plus rapidement que le sud. Le régime des précipitations serait également perturbé et le modèle anticipe une hausse légère dans la majeure partie du pays et une réduction dans les plaines du centre (figure 1).
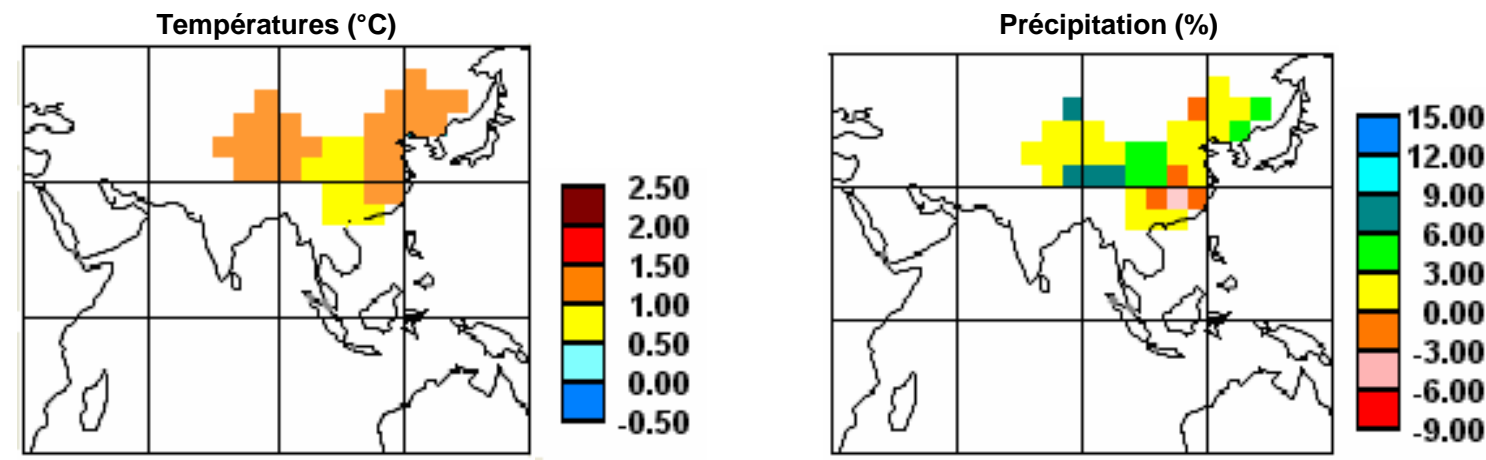

Figure 1 : Variations des températures et des précipitations en 2030 par rapport à 1990, scénario A1B.

Source : MAGICC-SCENGEN

\footnotetext{
${ }^{1}$ IPCC, Climate Change 2007, The Physical Science Basis, Summary for the Policymakers, Contribution of Working Group I to the Fourth Assessment Report of the Intergovernmental Panel on Climate Change, Genève, 2007.

${ }^{2}$ Mike Hulme et al., Using a Climate Scenario Generator for Vulnerability and Adaptation Assessments: MAGICC and SCENGEN Version 2.4, Climatic Research Unit, UEA, Norwich, 2000.
} 
L'analyse des enjeux du changement climatique nécessite de prendre pour point de départ les risques liés à la variabilité climatique et la gestion des ressources naturelles et d'analyser comment les modifications attendues du climat pourraient les amplifier. En Chine, la situation qui caractérise les ressources hydriques dans les plaines du nord est au cœur des problématiques de développement. L'équilibre entre les disponibilités et les usages de l'eau est déjà rompu et cette tendance pourrait encore être accentuée par le changement climatique. La rareté croissante de l'eau et la concurrence de l'industrie et des villes imposent une grande pression sur les capacités d'irrigation de l'agriculture. La production de céréales, secteur extrêmement dépendant de l'irrigation, fait ainsi face à un défi sans précédent.

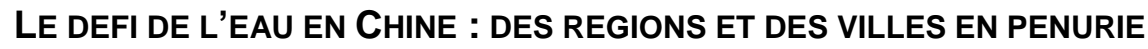

\section{Des ressources limitées, inégalement distribuées et surexploitées}

A première vue, la Chine est un pays relativement bien doté en eau. Les ressources mobilisables sont estimées à 2812 milliards de mètres cubes par an, plaçant la Chine au sixième rang mondial. Cependant, ramenées à la population, les disponibilités ne sont que de 2200 mètres cubes par habitant et par an, l'un des niveaux les plus bas du monde, mais qui constitue toutefois une dotation supérieure au seuil de « stress hydrique $^{3} »$. Toutefois, ce sont les grandes disparités régionales qui illustrent le mieux la gravité de la question de l'eau en Chine. Si la demande est croissante dans l'ensemble du pays, les disponibilités dans le nord (757 mètres cubes par habitant) représentent seulement un quart de celles du sud (3 208), ce qui place l'ensemble de la région du nord dans une situation de "pénurie d'eau ». La Chine du nord, qui abrite $42 \%$ de la population et surtout $60 \%$ des terres arables, ne possède que $14 \%$ des ressources hydriques chinoises. A l'échelle des bassins hydrographiques, les contrastes sont encore plus édifiants (graphique 1). Les taux d'utilisation des ressources dans les bassins du nord sont souvent trois à quatre fois supérieurs à ceux du sud et dépassent fréquemment les seuils de surexploitation. Ce taux excède notamment $95 \%$ dans le bassin de la rivière Hai.

\footnotetext{
${ }^{3}$ Les Nations unies et la Banque mondiale considèrent qu'un pays est en «stress hydrique » lorsque les ressources disponibles annuelles par tête sont inférieures ou égales à 2000 mètres cubes et en " pénurie d'eau » lorsqu'elles sont inférieures ou égales à 1 000. En dessous de 500, le pays est en "pénurie hydrique absolue ». Ces quantités incluent les usages domestiques mais également les consommations des activités productives.
} 


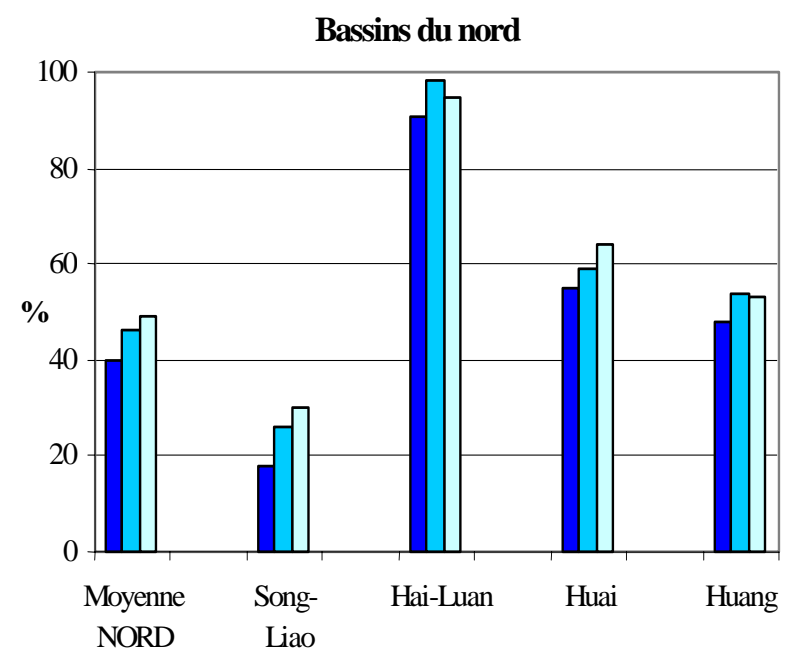

๑ $1980 \square 1993 \square 2000$

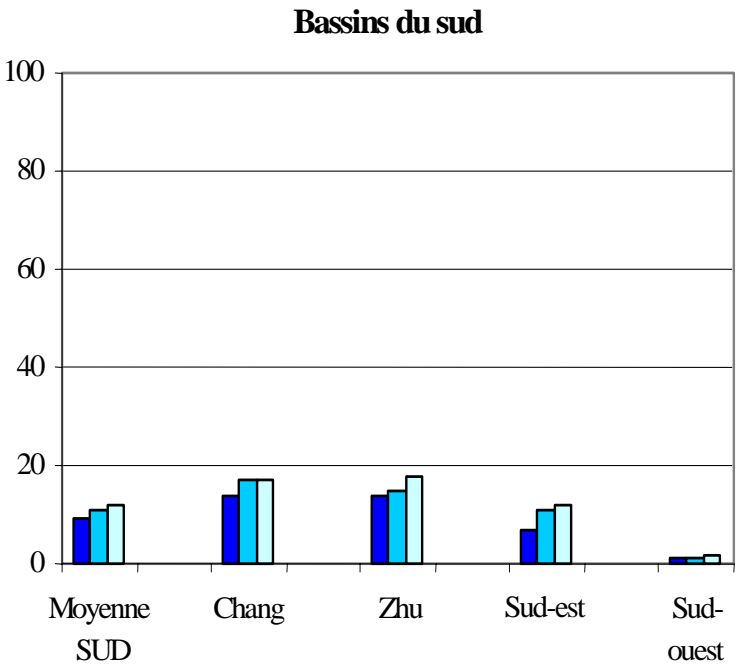

Graphique 1 : Taux d'utilisation des ressources hydriques dans les principaux bassins hydrographiques chinois

Source : Chinese Statistical Yearbook, 2003

Face à ces ressources limitées, on assiste à une augmentation significative de la demande globale, drainée principalement par l'industrialisation et l'urbanisation. L'irrigation des cultures reste le principal poste d'utilisation des ressources hydriques en concentrant $67 \%$ des consommations, suivi par l'industrie qui compte aujourd'hui pour $21 \%$ de la demande ${ }^{4}$. Mais l'eau utilisée par l'agriculture n'augmente que de manière marginale depuis 1980, alors même que la Chine relance depuis quelques années son programme d'autosuffisance alimentaire. Par contre, avec la croissance économique considérable et l'industrialisation accélérée qu'a connues la Chine, les consommations de l'industrie ont été multipliées par trois. La croissance des usages domestiques est également très largement dominée par les besoins des villes. Les consommations s'accroissent fortement à mesure que les ménages urbains, qui intègrent progressivement la classe moyenne, adoptent les standards de consommation qui la caractérisent.

En dépit des pénuries, l'utilisation des ressources hydriques est relativement inefficace. La pollution observée dans la plupart des cours d'eau chinois contribue également à accentuer la rareté de la ressource. Selon la SEPA ${ }^{5}, 34 \%$ des eaux des sept principaux cours d'eau chinois sont fortement polluées. La rivière des Perles et le Yangtze affichent une bonne qualité globale, mais on ne peut pas en dire autant de la plupart des cours d'eau du nord du pays qui souffrent d'une pollution chronique, la situation de la rivière Hai étant particulièrement alarmante.

La rareté de l'eau est particulièrement marquée dans le bassin des «3-H ». Cette région regroupe les trois principaux cours d'eau de la Chine du nord, le Huanghe (fleuve Jaune) et les rivières Hai et Huai. Elle couvre les villes de Pékin et Tianjin ainsi que les principales provinces agricoles chinoises. Le bassin des 3$\mathrm{H}$, véritable "grenier à céréales » de la Chine, concentre $39 \%$ des terres arables chinoises, abrite 460 millions d'habitants, génère un tiers du PIB national, mais ne détient que $10 \%$ des ressources en eau du pays. La Banque mondiale y estimait le déficit hydrique à déjà 39 milliards de mètres cubes en 2000 et les disponibilités moyennes par habitant à seulement 525 mètres cubes par an, bien en dessous donc des

\footnotetext{
${ }^{4}$ World Bank, China Agenda for Water Sector Strategy for North China, Main Report, Washington DC, 2002

${ }^{5}$ SEPA (State Environmental Protection Administration), Report on the State of the Environment in China, 2005.
} 
seuils de pénurie. Les pénuries apparaissent également dans les centres urbains en croissance rapide. On estime que sur les 600 villes que compte la Chine, 400 subissent des pénuries, et parmi ces dernières, 110 connaissent de sérieux déficits. Elles se concentrent surtout au nord du pays.

Pour compenser les déficits des eaux de surface, les ressources souterraines sont de plus en plus sollicitées dans le nord du pays et les aquifères sont souvent consommés à des rythmes supérieurs à ceux de leur reconstitution. En 1995, on estimait que les eaux souterraines fournissaient près de $60 \%$ de la demande des zones urbaines de la région des 3-H et chaque année Pékin puise $75 \%$ de ses consommations dans des aquifères non renouvelables. La conséquence de cette surexploitation est que les niveaux des nappes souterraines ont diminué de 50 à 90 mètres dans les plaines de la rivière Hai et de 100 à 300 mètres dans la région de Pékin, causant des problèmes d'intrusions salines dans les nappes côtières et des dommages sévères aux constructions à Pékin, Tianjin et Shanghai.

\section{Les effets de l'accentuation des sécheresses}

La rareté structurelle de l'eau est donc la conséquence de l'inégale répartition des ressources entre le nord et le sud, de l'importance de la population et du processus accéléré d'urbanisation et d'industrialisation. Elle est également le résultat des tendances du climat observées ces dernières décennies. Les données de longue période montrent que les températures augmentent continuellement depuis les années 1960 avec pour conséquence une augmentation de l'évaporation. En même temps, les précipitations présentent une tendance à la baisse, notamment dans les plaines du nord ${ }^{6}$. Ces modifications expliquent en partie les diminutions parfois dramatiques des écoulements de surface.

Un élément majeur qui alimente la crise hydrique est l'occurrence des sécheresses. Depuis les années 1980, la fréquence, la magnitude et la durée des sécheresses ont été croissantes, causant des dommages sévères. La Chine a été durement touchée ces dix dernières années et les plaines du Nord ont subi sept années consécutives de sécheresse, un record depuis l'avènement de la république populaire de Chine. Les années 2000, 2002 et 2006 ont vu les pires sécheresses depuis 50 ans.

La multiplication des sécheresses et l'augmentation des prélèvements conduisent à des situations particulièrement alarmantes comme celle du fleuve Jaune, berceau de la civilisation Han et cœur du principal bassin céréalier, qui fait figure aujourd'hui d'un "vieillard épuisé ». Les écoulements diminuent depuis 30 ans $^{7}$ et le fleuve s'est asséché chaque année entre 1985 et 2000 avant d'atteindre la mer Jaune (graphique 2). Les durées d'assèchement s'allongent et l'aire géographique concernée s'étend : en 1996, pour la première fois, les eaux du Huanghe n'ont pas atteint la province du Shandong. En 1997, la situation a été aggravée par la sécheresse et elles ont à peine atteint la province du Hebei. Cette année-là, l'assèchement a duré 227 jours contre 40 au début des années 1990, un record ${ }^{8}$.

\footnotetext{
${ }^{6}$ Jianping Yang et al., " Fluctuations of the Semi-Arid Zone in China and Consequences for Society », Climatic Change, 72 (1-2)[PAS CLAIR] , , 2005, p. 171-188.

${ }^{7}$ YRCC (Yellow River Conservancy Commission), Yellow River in the 20th Century, Yellow River Water Utilization Publisher, 2001.

${ }^{8}$ Jean-Pierre Cabestan, "Vers une crise alimentaire en Chine et dans le monde », interview de Lester Brown, Perspectives Chinoises, $\mathrm{n}^{\circ} 18,1997$, p. 1.
} 
Evolution des précipitations

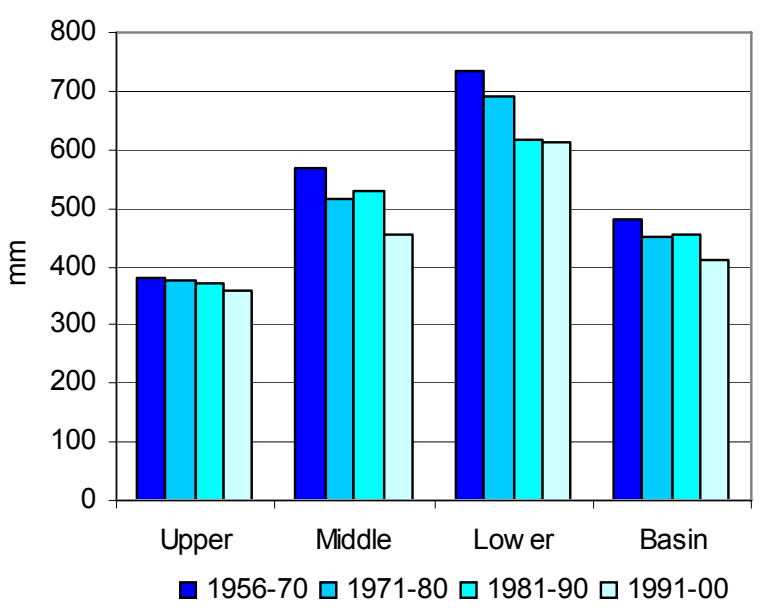

Evolution des écoulements

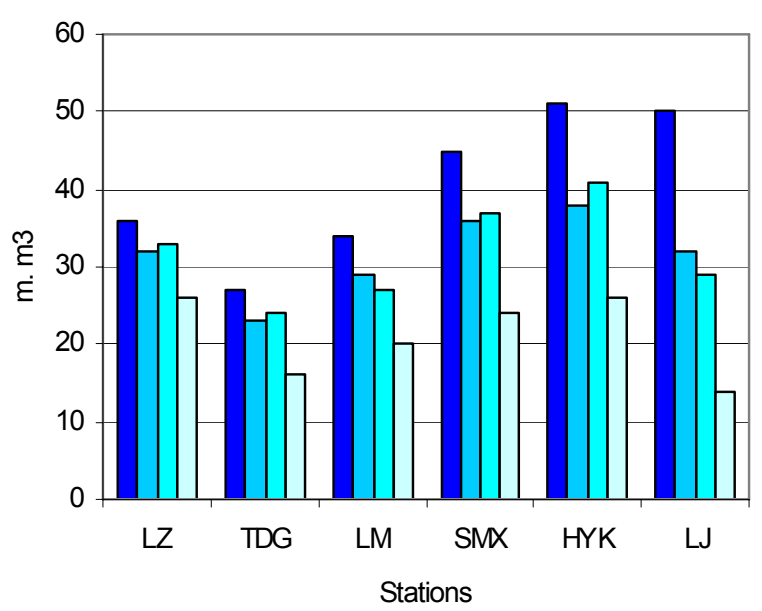

LZ = Lanshou, $T D G=$ Toudaoguai, $L M=$ Longmen, SMX = Sanmenxia, HYK, Huayuankou, LJ = Lijin

Graphique 2 : Evolution des précipitations et des écoulements dans le bassin du fleuve Jaune

Source : Yellow River Conservancy Commission, 2002

Les déficits hydriques sont donc alarmants dans le nord de la Chine et de nombreuses preuves montrent que la situation empire depuis 20 ans, en relation avec le développement économique, l'urbanisation et l'amplification des sécheresses. La rareté de l'eau est particulièrement coûteuse pour le développement économique et social du pays. Ainsi, la sécheresse qui a frappé la Chine en 2002 a affecté 65 millions de personnes et a coûté 1,2 milliard de dollars ${ }^{9}$.

\section{HORIZON 2030 : DES RISQUES AMPLIFIES PAR LE CHANGEMENT CLIMATIQUE}

\section{Des déficits hydriques tendanciels...}

Le problème de la sécurité hydrique des régions du nord est un enjeu de développement prioritaire pour la Chine. L'analyse des tendances de l'utilisation de l'eau et des capacités à augmenter l'offre pour les prochaines décennies montre que les déficits hydriques vont s'intensifier, et pourraient encore être aggravés par le changement climatique. Le cas des plaines du Nord et l'avenir du bassin des 3-H donnent une image claire du défi que devra affronter la Chine entre 2000 et 2050.

Selon les projections de la Banque mondiale ${ }^{10}$, la demande d'eau totale dans cette région augmentera de $20 \%$ entre 2000 et $2050^{11}$, pour atteindre un volume de 200 milliards de mètres cubes. La hausse des besoins particulièrement forte entre 2000 et 2020, de l'ordre de $7 \%$ par décennie, est essentiellement due aux besoins domestiques et industriels des zones urbaines qui doivent augmenter respectivement de $66 \%$ et $56 \%$ entre 2000 et 2020 . Concernant l'offre, les capacités additionnelles sont très limitées dans le bassin des 3-H indiquant que toutes les possibilités sont déjà plus ou moins utilisées à leur maximum. L'offre dans cette région pourrait vraisemblablement augmenter de seulement dix milliards de mètres cubes d'ici 2020 pour ensuite se stabiliser rapidement.

\footnotetext{
${ }^{9}$ EM-DAT, The International Disaster Database, http://www.em-dat.net/

${ }^{10}$ World Bank, China Agenda for Water Sector Strategy for North China, op. cit.

${ }^{11}$ Ces projections supposent une amélioration de l'efficacité de l'irrigation de $8 \%$ et une augmentation des prix de l'eau de $2 \%$ par an.
} 
Le déficit hydrique dans le bassin des 3-H doit s'approfondir lourdement. Déjà estimé à 39 milliards de mètres cubes en 2000, il doit augmenter de $25 \%$ d'ici 2020 et de $46 \%$ à l'horizon 2050, pour atteindre alors 57 milliards de mètres cubes. Kaiyuan Jieliu, qui signifie littéralement «ouvrir de nouvelles sources et économiser sur l'utilisation », est un adage bien connu pour la gestion des ressources hydriques en Chine. L'introduction de mesures supplémentaires d'adaptation à la rareté permettrait de réduire l'écart entre les besoins et les disponibilités ${ }^{12}$. Du côté de la demande, l'augmentation du prix de l'eau de $10 \%$ par an et l'amélioration de l'efficacité de l'irrigation de $10 \%$ supplémentaires par rapport au scénario de référence réduiraient le déficit hydrique de $14 \%$ en 2020 et de $22 \%$ en 2050. Du côté de l'offre, le recyclage des eaux usées améliorerait encore la situation de $4 \%$, alors que la mise en place des transferts interrégionaux du sud vers le nord réduirait les déficits hydriques de $22 \%$ supplémentaires. Ces quatre mesures prises ensemble diminueraient donc l'écart entre l'offre et la demande d'eau de $39 \%$ en 2020. Toutefois, en dépit de l'ampleur des efforts qu'impliquerait un tel scénario, les déficits resteraient conséquents (graphique 3).
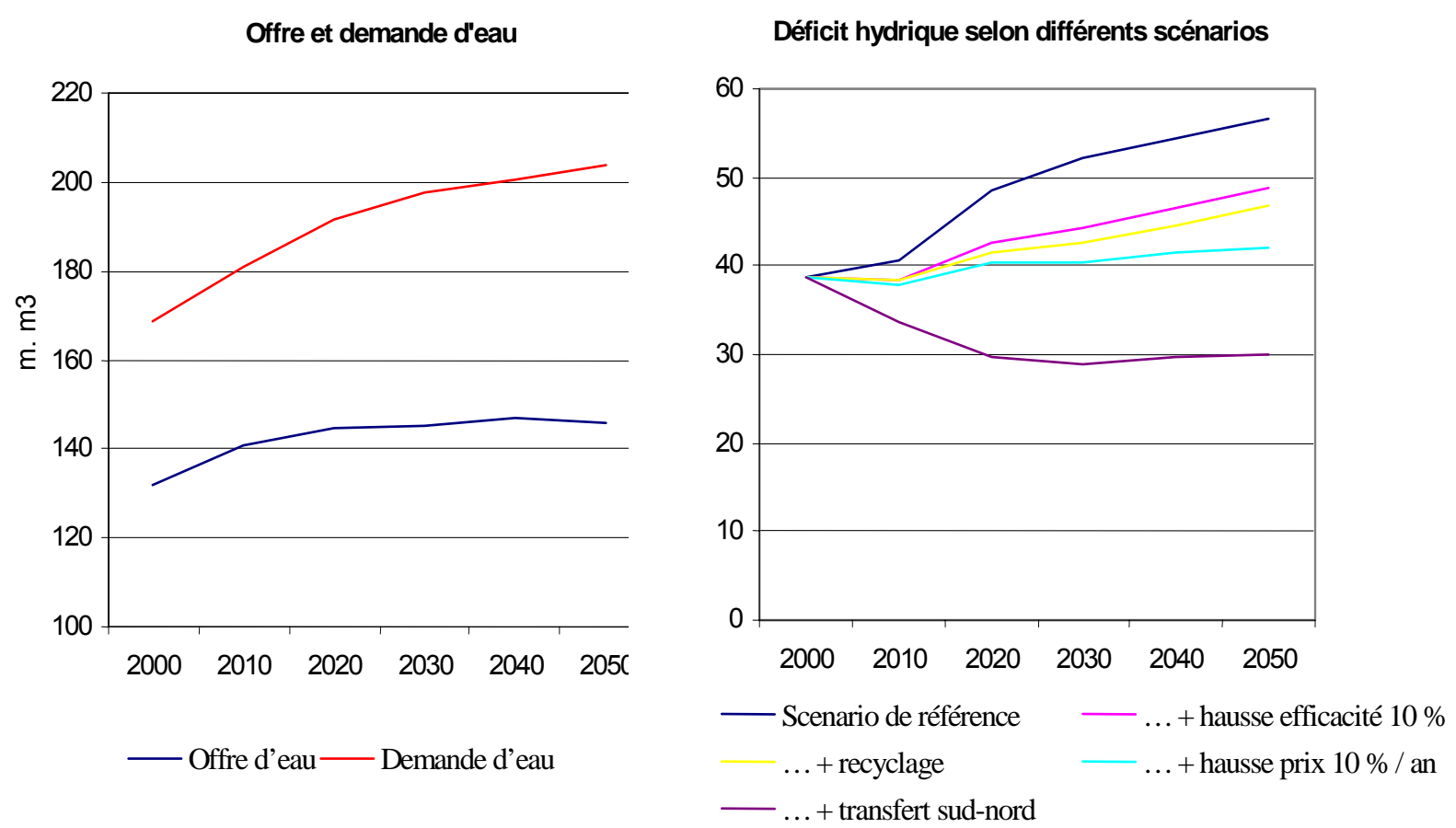

Graphique 3 : Tendances de l'offre et la demande d'eau et des déficits hydriques dans le bassin des 3-H

Source : Banque mondiale, 2002

\section{... amplifiés par le changement climatique}

Les déficits hydriques tendanciels subis par le nord de la Chine pourraient être amplifiés par le changement climatique. Les modifications des températures et du régime des précipitations attendues à l'horizon 20302050 doivent altérer la saisonnalité et la magnitude des débits des cours d'eau, avec de fortes implications sur les ressources hydriques et leur gestion.

L'adaptabilité des ressources hydriques au changement climatique dépend de leur vulnérabilité et de leur sensibilité aux modifications du climat. Au regard des taux de prélèvement, de la surexploitation des

\footnotetext{
${ }^{12}$ World Bank, China Agenda for Water Sector Strategy for North China, op. cit.
} 
aquifères profonds et de la variabilité des écoulements, les systèmes hydriques du nord de la Chine apparaissent de plus en plus vulnérables. Les marges de manœuvre sont donc généralement faibles. La sensibilité des ressources au changement climatique est croissante du sud humide vers le nord semi-aride : de faibles variations des précipitations ou des températures impliqueraient d'importantes conséquences sur les ressources hydriques. Les résultats des modèles montrent que les débits des cours d'eau diminueront significativement dans de nombreux bassins du nord et augmenteront dans le nord-est et le sud du pays ${ }^{13}$. Les bassins des rivières Huai et Huang apparaissent particulièrement à risque puisque l'on anticipe une réduction des débits de $11 \%$ et $6 \%$ respectivement. La région de Pékin-Tianjin-Tangshan, déjà fortement contrainte par les disponibilités en eau, ne serait pas non plus épargnée (tableau 1).

Tableau 1 : Variations attendues des températures, des précipitations, de l'évaporation et des écoulements dans quatre bassins du nord de la Chine

\begin{tabular}{lc|c|c|c|c}
\hline Bassins & & Liao & $\begin{array}{c}\text { Pékin-Tianjin- } \\
\text { Tangshan }\end{array}$ & Huanghe & Huaihe \\
\hline \multirow{2}{*}{$\begin{array}{c}\text { Scénario } \\
\text { climatique }\end{array}$} & $\Delta \mathrm{T}\left({ }^{\circ} \mathrm{C}\right)$ & 1,2 & 1,2 & 1,2 & 1,1 \\
\cline { 2 - 6 } & $\Delta \mathrm{P}(\%)$ & 3,9 & 1,1 & 0,3 & 0,1 \\
\hline $\begin{array}{c}\text { Réponse des } \\
\text { variables } \\
\text { hydriques }\end{array}$ & $\Delta \mathrm{E}(\%)$ & 1,2 & 3,8 & 2,5 & 3,9 \\
\cline { 2 - 6 } & $\Delta \mathrm{Ec}(\%)$ & 6,9 & $-4,4$ & $-5,9$ & -11 \\
\hline
\end{tabular}

Source : Ying Aixen, « Impact of Global Climate Change on China's Water Resources », op. cit.

En plus de ces modifications moyennes des débits, le changement climatique doit augmenter la fréquence et la magnitude des événements météorologiques extrêmes comme les sécheresses. La modification du régime des sécheresses est un aspect essentiel dans l'évaluation des risques, ces événements ayant déjà des impacts socioéconomiques sévères. Dans le bassin des $3-\mathrm{H}$, les années sèches impliquent une augmentation des déficits hydriques de l'ordre de $75 \%$ par rapport aux années normales, déficits causés à la fois par une baisse de l'offre d'eau annuelle de 5,5\% et par une augmentation de la demande tirée par les besoins de l'irrigation de l'ordre de $15 \%{ }^{14}$. Au regard des impacts des sécheresses des 15 dernières années, on peut comprendre que les effets du changement climatique sur la fréquence de tels événements dans les décennies à venir posent des risques énormes pour la gestion du secteur hydrique et la satisfaction des besoins.

UNE AGRICULTURE PARTICULIEREMENT VULNERABLE AU CHANGEMENT CLIMATIQUE

\section{Les enjeux de l'eau pour l'agriculture du nord de la Chine}

\footnotetext{
${ }^{13}$ Ying Aixen, Impact of Global Climate Change on China's Water Resources, Environmental Monitoring and Assessment, 61(1), 2000, p. 187-191.

${ }^{14}$ World Bank, China Agenda for Water Sector Strategy for North China, op. cit.
} 
La rareté croissante de l'eau et la concurrence des autres secteurs imposent une grande pression sur les capacités d'irrigation des régions du Nord. La production de céréales, secteur extrêmement dépendant de l'irrigation, fait ainsi face à un défi sans précédent. Dans le bassin des $3-\mathrm{H}$, l'agriculture est le principal consommateur d'eau, mais les pénuries sont également essentiellement supportées par ce secteur puisqu'il concentre plus de $80 \%$ du déficit total de la région. Chaque année, c'est plus d'un quart de la demande d'origine agricole qui n'est pas satisfaite. Lors des années de sécheresse, les déficits des cultures sont quasiment doublés et atteignent 62 milliards de mètres cubes. L'agriculture, qui voit ses besoins augmenter durant les années sèches alors que les disponibilités diminuent, joue finalement un rôle de tampon pour garantir la satisfaction des besoins des autres secteurs. Ces années là, l'agriculture est souvent sacrifiée pour limiter l'augmentation des déficits hydriques subis par l'industrie et les ménages.

L'insuffisance structurelle des apports en eau et la fréquence accrue des sécheresses réduisent fortement les potentiels agricoles. En année moyenne, les modèles estiment que les pénuries réduisent la production annuelle de grains de $15 \%$ dans le bassin des $3-\mathrm{H}$, soit 20 millions de tonnes. Cette amputation du potentiel céréalier dépasse $20 \%$ dans le bassin de la rivière Hai. Les ressources hydriques fournies pour l'irrigation stagnent depuis 20 ans alors même que la surface irriguée a crû de près de $17 \%$, indiquant un déclin des disponibilités hydriques à l'hectare ${ }^{15}$. Cette situation défavorable associée à une récurrence accrue d'années sèches a conduit à une dégradation de la production céréalière durant cinq années consécutives. Entre 1999 et 2003 , les récoltes ont chuté de $17,5 \%$ et sur la seule année 2000 , où la sécheresse a été particulièrement marquée, la production de grains a diminué de $10,5 \%{ }^{16}$. La sensibilité forte aux conditions hydriques et climatiques implique que ce secteur pourrait être l'un des plus sévèrement touchés par le réchauffement du climat.

\section{Vulnérabilité et sensibilité agricole}

Le contexte agricole montre que de nombreuses régions du nord de la Chine seraient à la fois très sensibles et très vulnérables au changement climatique. Les modifications attendues des températures et des précipitations pour les prochaines décennies impliquent en effet une augmentation des besoins d'irrigation dans un contexte de réduction des ressources disponibles. L'augmentation des épisodes de sécheresse amplifierait lourdement ces tendances. Au niveau global, le changement climatique doit conduire à un déplacement des étages agroclimatiques vers le nord de la Chine posant ainsi un défi aux régions semiarides de la région des 3-H. Les impacts sur les principales zones de production céréalière montrent un effet globalement négatif sur les rendements ${ }^{17}$.

Les modèles agroclimatiques anticipent que la conjonction des modifications des températures, des précipitations et de l'évapotranspiration augmentera les déficits d'humidité des sols pour les cultures de 20 à $60 \mathrm{~mm}$ dans les plaines du nord, alourdissant d'autant les besoins d'irrigation ${ }^{18}$. Quatre régions situées dans

\footnotetext{
${ }^{15}$ Hong Yang, Zehner A., "China's Regional Water Scarcity and Implications for Grain Supply and Trade », Environment and Planning $A, \mathrm{n}^{\circ} 33,2001$, p. 79-95.

${ }^{16} \mathrm{FAO}$, http://faostat.fao.org, 2006

${ }^{17}$ Ana Iglesias et al., "Climate Change in Asia: A Review of the Vulnerability and Adaptation of Crop Production », Water, Air, and Soil Pollution, $\mathrm{n}^{\circ}$ 92, p. 13-27, 1996.

${ }^{18}$ Fulu Tao et al., "Future Climate Change, the Agricultural Water Cycle, and Agricultural Production in China », Agriculture, Ecosystems \& Environment, 95(1), 2003, p. 203-215.
} 
en Chine du nord particulièrement sensibles au changement climatique risquent de tomber en dessous de certains seuils hydriques critiques : la région longeant la Grande Muraille, les plaines du Huanghe et de la rivière Hai, le nord de la rivière Huai et le plateau du Loess. Ces régions devraient souffrir d'un risque accru de sécheresse, d'une amplification des déficits hydriques et de problèmes de désertification. Les indices de vulnérabilité basés sur le contexte agricole et les capacités socioéconomiques à s'adapter montrent que parmi les 30 provinces chinoises, le Shanxi, la Mongolie intérieure, le Gansu, le Hebei et le Ningxia seraient particulièrement vulnérables et les moins aptes à s'adapter au changement climatique ${ }^{19}$. Dans le nord de la Chine, les régions les plus sensibles sont donc aussi les plus vulnérables et concernent la majeure partie du bassin des $3-\mathrm{H}$.

\section{Un contexte de compétition hydrique intersectorielle défavorable}

Le contexte de compétition intersectorielle pour l'eau dans lequel s'inscrit le réchauffement climatique est essentiel pour anticiper les risques qui pèsent sur l'agriculture. Dans la région des $3-\mathrm{H}, 80 \%$ des terres cultivées sont irriguées et ces apports d'eau constituent un facteur indispensable au système de doubles récoltes annuelles qui caractérise la région et qui sous-tend son statut de "grenier à céréales". L'agriculture est le principal consommateur d'eau mais n'est pas un secteur prioritaire. L'allocation de l'eau favorise de manière croissante les besoins industriels et domestiques, notamment pendant les périodes de sécheresse. Dans ce contexte, les réductions des disponibilités hydriques causées par le changement climatique font peser un double fardeau sur les capacités agricoles : l'agriculture aura d'autant plus besoin d'eau que les conditions seront sèches, et dans le même temps la réduction de l'offre implique qu'une part d'autant plus faible des ressources hydriques disponibles sera allouée à l'agriculture. Les variations des modes d'irrigation selon les conditions climatiques montrent bien les enjeux : l'occurrence des sécheresses réduit la part des terres cultivées en irrigation totale de 20 points et augmente la part en culture pluviale et en irrigation partielle de 13,5 et 6,5 points respectivement (graphique 4). Dans la mesure où le changement climatique multiplierait la fréquence des années sèches, les risques pesant sur les capacités d'irrigation sont énormes.

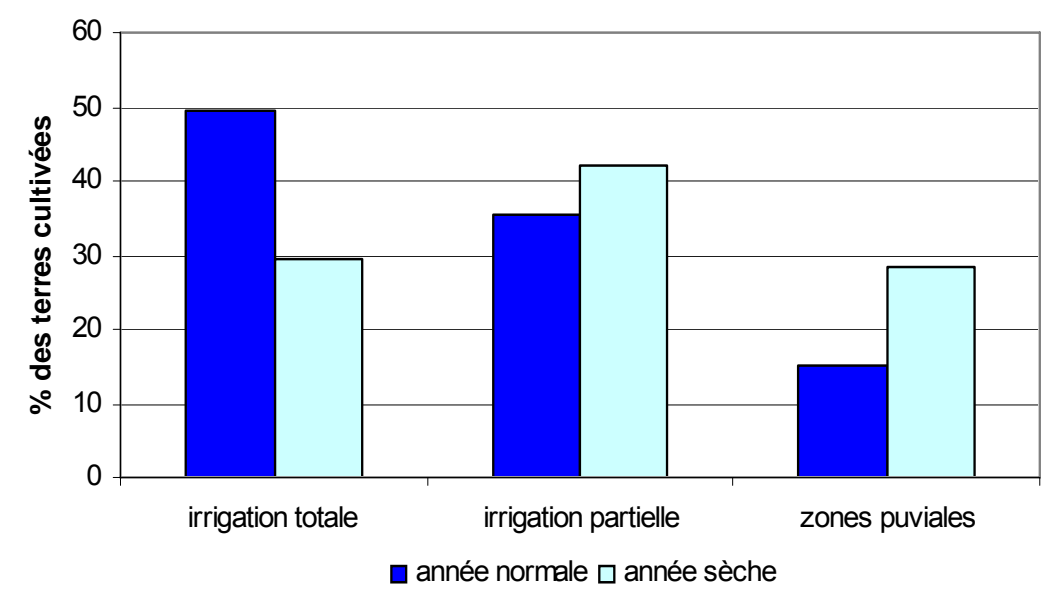

Graphique 4 : Répartition des modes d'irrigation dans le bassin des 3-H selon les conditions climatiques, horizon 2030

Source : World Bank, China Agenda for Water Sector Strategy for North China, op. cit.

\footnotetext{
${ }^{19}$ Lin Erda, «Agricultural Vulnerability and Adaptation to Global Warming in China », Water, Air, and Soil Pollution, n 92, p. 63-73, 1996.
} 
Des politiques d'adaptation de l'offre et de la demande d'eau sont envisageables dans le nord du pays, mais il est clair que l'agriculture devra compter essentiellement sur ses propres capacités à économiser des ressources. Le potentiel d'économie d'eau dans ce secteur n'est pas négligeable à court terme, mais pourrait néanmoins être restreint par le changement climatique. Pour ce qui est du transfert sud-nord, principal apport d'eau pour le futur dans le nord de la Chine, le projet ne porte pas à l'optimisme car, à l'instar de tous les projets à grande échelle en construction ou planifiés, il n'aurait pas vocation à augmenter l'irrigation ${ }^{20}$. Dans le meilleur des cas, l'agriculture ne recevrait qu'une partie résiduelle des transferts, la priorité étant invariablement donnée aux besoins urbains ${ }^{21}$. La position résiduelle de l'irrigation dans l'allocation de l'eau semble inévitable : le rendement économique de chaque unité d'eau consommée par l'agriculture est faible par rapport aux autres secteurs, et le faible prix de l'eau d'irrigation par rapport aux usages industriels et domestiques incite les administrations locales à détourner l'eau de l'agriculture vers d'autres secteurs $^{22}$. Dans ce contexte, le changement climatique pourrait aggraver les conflits intersectoriels sur l'eau qui prennent déjà parfois une tournure violente ${ }^{23}$.

\section{Impacts sur les rendements agricoles}

Les modèles estiment que le changement climatique aura un effet globalement négatif sur le potentiel agricole chinois bien que de nouvelles zones pourraient devenir aptes à l'agriculture dans l'extrême nord du pays. Les trois principales cultures que sont le blé, le riz et le maïs doivent voir leurs rendements se restreindre de $10 \%$ en moyenne durant la première moitié $\mathrm{du} X X \mathrm{Xl}^{\mathrm{e}}$ siècle. L'augmentation des températures doit raccourcir les durées de croissance des cultures et la qualité nutritionnelle des céréales mais l'agriculture sera principalement touchée par la hausse des sécheresses et des déficits hydriques. On anticipe que les rendements de blé diminueraient de $4 \%$ en moyenne dans les zones irriguées à $16 \%$ en zones pluviales ${ }^{24}$. Les cultures de maïs seraient également affectées, les modèles estimant en effet que les récoltes chuteraient de $5 \%$. Ici encore, les cultures pluviales apparaissent beaucoup plus sensibles ${ }^{25}$. Enfin, concernant le riz, la productivité diminuerait graduellement de 6 à $17 \%$ du sud au nord ${ }^{26}$. L'augmentation de la variabilité climatique et des sécheresses accroîtrait encore la probabilité de faibles récoltes. Le changement climatique pourrait également aggraver les problèmes de désertification en raison de la hausse des températures et du caractère plus aléatoire des précipitations; il conduirait également à augmenter l'utilisation de fertilisants et de pesticides, non sans conséquence sur la qualité de l'eau et des sols.

\footnotetext{
${ }^{20}$ Hong Yang , Zehner A., "China's Regional Water Scarcity and Implications for Grain Supply and Trade », op. cit.

21 ZSN, Chinese Water Conservation Yearbook, Water and Electricity Publishing House, Pékin, 1997.

${ }^{22}$ Les bénéfices économiques des transferts interrégionaux par la route centrale sont estimés à 0,99 yuan le mètre cube d'eau dans les secteurs urbains et industriels, contre 0,56 dans l'agriculture. Dans ce même projet, le prix moyen de l'eau à destination de l'industrie serait de 0,31 yuan le mètre cube et pour l'agriculture de 0,06 yuan le mètre cube (ZSN, Chinese Water Conservation Yearbook, op. cit.)

${ }^{23}$ Par exemple, le 6 juillet 2000, des milliers de paysans de la plaine du nord se sont heurtés aux forces de police, leur colère ayant été provoquée par un projet gouvernemental qui allouait l'eau de certains réservoirs à des villes et à l'industrie plutôt qu'à l'irrigation.

${ }^{24}$ La prise en compte de l'effet fertiliseur du $\mathrm{CO}_{2}$ pourrait limiter fortement ces impacts, mais ils restent actuellement mal connus.

25 Whang Jinghua, Lin Erda, "The Impacts of Potential Climate Change and Climate Variability on Simulated Maize Production in China », Water, Air, and Soil Pollution, $\mathrm{n}^{\circ}$ 92, 1996, p. 75-85.

${ }^{26}$ UNFCCC National Communication, The People's Republic of China, Initial National Communication on Climate Change, 2004.
} 
Les impacts de la multiplication des sécheresses sur les modes de cultures pourraient être sévères lorsque I'on a à l'esprit la vulnérabilité des modes d'irrigation. Etant donné le faible rendement économique de la production céréalière par unité d'eau utilisée et l'augmentation de la rareté de la ressource dans le nord de la Chine, une réduction de la surface irriguée est à envisager. La baisse des capacités d'irrigation saisonnière pourrait mettre en péril le système de doubles récoltes annuelles. Etant donné le climat de mousson qui domine dans la région, les cultures d'hiver, quasiment intégralement tributaires de l'irrigation semblent particulièrement à risque.

Cette évolution du potentiel agricole de la Chine pose des risques économiques et sociaux importants pour les agriculteurs. L'augmentation de la probabilité de faibles récoltes pourrait en effet miner l'amélioration de leur niveau de vie alors même qu'ils apparaissent déjà comme les laissés-pour-compte du développement accéléré de la Chine. En particulier, la multiplication des sécheresses et les pertes de récoltes qui en découlent pourraient rapidement compromettre les capacités des agriculteurs à absorber ces chocs et les conduire à grossir les rangs de l'exode rural vers les centres urbains en plein essor ou vers d'autres régions agricoles plus favorables. Ces "réfugiés du climat " sont déjà une réalité et leur déplacement pourrait même être orchestré par le pouvoir central comme le montre la sécheresse de 2006, qui a vu le déplacement organisé de près de 30000 paysans de Chongqing et Chengdu vers le Xinjiang.

\section{Autosuffisance alimentaire ou importation d'eau virtuelle?}

Les risques posés par le changement climatique sur l'agriculture peuvent enfin être replacés dans le contexte de l'incertitude qui pèse sur la production céréalière future et les capacités de la Chine à satisfaire ses propres besoins. La question de la sécurité alimentaire et des impacts potentiels de l'entrée de la Chine sur les marchés agricoles internationaux est un sujet brûlant. En 1994, L. Brown dans un article intitulé «Who will feed China? » a posé le débat et suscité une véritable polémique. Son analyse est simple : les capacités décroissantes de la Chine à produire des céréales en raison de la perte de terres arables qui suit l'industrialisation et de l'augmentation des déficits hydriques, la conduira à importer des quantités énormes de céréales ${ }^{27}$. Les conséquences seraient une hausse sensible des prix sur les marchés internationaux et une menace croissante sur la sécurité alimentaire et la stabilité politique des pays en développement. Le changement climatique pourrait accélérer ces tendances en aggravant les déficits hydriques et en alourdissant les pertes de sols par l'amplification de la désertification.

Cette nécessité d'un recours massif aux marchés internationaux afin de pourvoir aux besoins en céréales, a jusqu'à présent été réfutée par les faits. Néanmoins, la baisse de la production observée entre 1999 et 2003 sans réelle augmentation des importations a été permise par des ponctions continuelles dans les stocks céréaliers constitués entre 1996 et 2002, comblant ainsi un manque annuel de 30 millions de tonnes. Si les capacités de production seront probablement réduites par le changement climatique, une crise agricole n'est probablement pas pour autant à attendre en Chine. Les effets sur les marchés mondiaux pourraient venir plus spécifiquement des stratégies d'adaptation qu'adoptera la Chine pour répondre à la crise hydrique. Les capacités physiques à produire les céréales nécessaires pour nourrir la Chine resteraient suffisantes, mais il

\footnotetext{
${ }^{27}$ L'analyse de Brown est cohérente avec d'autres études, de la Banque mondiale et de l'United State Department of Agriculture notamment.
} 
ne serait pas forcément rationnel de pousser l'utilisation des terres et des ressources hydriques à leur maximum ${ }^{28}$.

II pourrait en effet devenir économiquement efficace d'avoir recours aux marchés mondiaux et de prendre part au « commerce d'eau virtuel ${ }^{29}$ ", en important de l'eau sous forme de céréales. Actuellement, un mètre cube d'eau d'irrigation est nécessaire pour produire un kilo de céréales. Si dix millions de tonnes de céréales étaient importées plutôt que produites dans la plaine du Nord, c'est près de dix milliards de mètres cubes d'eau qui seraient économisés. Les ressources limitées en sols et en eau pourraient alors être utilisées de manière plus efficace en substituant par exemple des cultures à haute valeur ajoutée, éventuellement tournées vers l'exportation. Etant donné le désavantage comparatif croissant de la Chine dans la production de céréales et son intégration continuelle dans l'économie mondiale, l'augmentation des importations de céréales semble inévitable. L'importation d'eau virtuelle apparaît comme une stratégie pertinente non seulement car elle allègerait sensiblement le stress hydrique de la Chine du nord, mais également parce qu'elle serait conforme à la voie dans laquelle l'économie chinoise se dirige. Reste à savoir si la Chine du socialisme de marché acceptera de remettre en question le principe de l'autosuffisance alimentaire du régime maoïste.

II demeure que les incertitudes sont persistantes en ce qui concerne l'évolution de la capacité mondiale de production de céréales, en particulier dans le contexte du changement climatique. Les effets d'un telle politique sur les capacités des pays en développement les plus pauvres à s'approvisionner sur les marchés internationaux de céréales ne seront peut-être pas indolores.

\section{CONCLUSION}

Le changement climatique pose un défi sans précédent aux systèmes hydriques et agricoles du nord de la Chine. Si les équilibres sont souvent déjà rompus, le réchauffement attendu pourrait jouer un rôle d'accélérateur d'une dynamique déjà observable d'aggravation des déficits hydriques et de détérioration des terres arables. Au-delà, se profilent les risques d'inondation des villes côtières menacées par la montée du niveau de la mer et l'extension des zones infectées par certaines maladies à vecteurs comme la malaria. La mise en place de mesures d'adaptation anticipatives, qui dans le cas de l'agriculture consisteraient en une politique de l'eau axée de manière structurelle sur la rareté de la ressource, pourraient réduire ces risques tout en répondant à des enjeux actuels. Dans ce contexte, la communauté internationale doit, parallèlement à ses efforts de réduction des émissions de gaz à effet de serre, proposer une voie d'adaptation pour les pays en développement.

\footnotetext{
${ }^{28}$ G. K. Heilig, China Food, Can China Feed Itself?, IIASA, Laxenburg, 1999.

${ }^{29}$ T. Allan, Water, Peace and the Middle East : Negotiating Water in the Jordan Basin, IB Tauris, Londres, 1996.
} 